\title{
Estratégias de cultura de paz na educação infantil sob a ótica de educadores
}

\section{Strategies of peace culture in childhood education under the educators 'views}

Estrategias para una cultura de paz en la educación infantil desde la perspectiva de los educadores

Rita de Cássia Ramires da Silva ORCID: https://orcid.org/0000-0002-0503-4299

Universidade Federal de Alagoas, Brasil

E-mail: rita.silva@esenfar.ufal.br

Adrielly Cristina de Lima Raimundo

ORCID: https://orcid.org/0000-0001-7290-8382

Universidade Federal de Alagoas, Brasil

E-mail: adrielly1322@hotmail.com

Camila Thayná Oliveira dos Santos

ORCID: https://orcid.org/0000-0002-9211-4595

Universidade Federal de Alagoas, Brasil

E-mail: camila.thay7@gmail.com

Lays da Silva Fidelis Freire

ORCID: https://orcid.org/0000-0003-1954-8458

Universidade Federal de Alagoas, Brasil

E-mail: laysfidelisfreire@gmail.com

Lindynês Amorim de Almeida

ORCID: https://orcid.org/0000-0001-9749-7938

Universidade Federal de Alagoas, Brasil

E-mail: lindyalmeida7@gmail.com

Rillary Caroline de Melo Silva

ORCID: https://orcid.org/0000-0002-1488-1887

Universidade Federal de Alagoas, Brasil

E-mail: melorillary@gmail.com

Jislene dos Santos Silva

ORCID: https://orcid.org/0000-0002-9917-9869

Universidade Federal de Alagoas, Brasil

E-mail: jih1995silva@gmail.com

Vitória Braz de Almeida

ORCID: https://orcid.org/0000-0002-9081-4037 Universidade Federal de Alagoas, Brasil

E-mail: vitoriabrazdealmeida24@gmail.com

Milena Alicia da Silva Santos

ORCID: https://orcid.org/0000-0002-8377-8680

Universidade Federal de Alagoas, Brasil E-mail: milenaalicia123@hotmail.com

Letícia de Macedo Santos

ORCID: https://orcid.org/0000-0002-3711-4170 Universidade Federal de Alagoas, Brasil

E-mail: leticiademacedosantos@gmail.com

Barbara Vitória dos Santos Torres

ORCID: https://orcid.org/0000-0002-0153-1554 Universidade Federal de Alagoas, Brasil E-mail: lucyleedean@gmail.com

Ana Carolina Santana Vieira

ORCID: https://orcid.org/0000-0002-7273-1414 Universidade Federal de Alagoas, Brasil E-mail: ana.vieira@eenf.ufal.br

Ingrid Martins Leite Lúcio

ORCID: https://orcid.org/0000-0003-2738-7527 Universidade Federal de Alagoas, Brasil

E-mail: ingridmll@esenfar.ufal.br

Rossana Teotônio de Farias Moreira

ORCID: https://orcid.org/0000-0002-0881-1997 Universidade Federal de Alagoas, Brasil

E-mail: rossanateo@hotmail.com

Anne Laura Costa Ferreira

ORCID: https://orcid.org/0000-0002-6803-3621 Universidade Federal de Alagoas, Brasil E-mail:annelaura1@hotmail.com 


\begin{abstract}
Resumo
A promoção da Cultura de Paz é um dos componentes essenciais à saúde da criança e sua abordagem para a educação infantil. A partir de desafios sociais observados em atividades de ensino, pesquisa e extensão universitárias, surgiu o objetivo de conhecer a percepção de educadores sobre estratégias de cultura de paz na educação infantil. Estudo do tipo qualitativo, realizado em um Centro Municipal de Educação Infantil, em área de vulnerabilidade social do município de Maceió-AL, no período de dezembro de 2019 a março de 2020, com nove profissionais da educação infantil, por meio das tecnologias de Grupos Focais para avaliação dos aspectos relacionados à Cultura de Paz. A partir dos encontros, evidenciou-se que as falas convergiram para três categorias: o conceito da cultura de paz, o trabalho com a cultura de paz na escola e a construção de estratégias de cultura de paz. A escassez de estudos e a dificuldade de compreensão sobre o tema, assim como a importância de integrar educação e a promoção da cultura de paz, foram aspectos que resultaram na sugestão de construção de um jogo de tabuleiro para subsidiar a resolução de conflitos de forma não violenta. $\mathrm{O}$ estudo apontou contribuições para a promoção da Cultura de Paz e estratégias lúdicas para o contexto da Educação Infantil, bem como a necessidade de outras pesquisas para exploração da temática.
\end{abstract}

Palavras-chave: Direitos humanos; Educação infantil; Cultura de paz.

\begin{abstract}
Promoting the Culture of Peace is one of the essential components of child health and its approach to early childhood education. From the social challenges observed in teaching, research and university extension activities, the objective of knowing the perception of educators about strategies for a culture of peace in early childhood education emerged. Qualitative study, carried out in a Municipal Center for Early Childhood Education, in an area of social vulnerability in the city of Maceió-AL, from December 2019 to March 2020, with nine professionals in early childhood education, using Group technologies Focal for the evaluation of aspects related to the Culture of Peace. From the meetings, it was evident that the speeches converged into three categories: the concept of the culture of peace, working with the culture of peace at school and the construction of culture strategies of peace. The scarcity of studies and the difficulty in understanding the theme, as well as the importance of integrating education and the promotion of a culture of peace, were aspects that resulted in the suggestion of building a board game to support conflict resolution in a non- violent. The study pointed out contributions to the promotion of the Culture of Peace and playful strategies for the context of Early Childhood Education, as well as the need for further research to explore the theme.
\end{abstract}

Keywords: Human rights; Child rearing; Culture of piece.

\title{
Resumen
}

La promoción de la cultura de paz es uno de los componentes esenciales de la salud infantil y su enfoque de la educación de la primera infancia. A partir de los desafíos sociales observados en las actividades de docencia, investigación y extensión universitaria, surgió el objetivo de conocer la percepción de los educadores sobre las estrategias para una cultura de paz en la educación infantil. Estudio cualitativo, realizado en un Centro Municipal de Educación Infantil, en un área de vulnerabilidad social de la ciudad de Maceió-AL, de diciembre de 2019 a marzo de 2020, con nueve profesionales en educación infantil, utilizando tecnologías del Grupo Focal para la evaluación de aspectos relacionados con la Cultura de Paz. De los encuentros se evidenció que los discursos convergieron en tres categorías: el concepto de cultura de paz, el trabajo con la cultura de paz en la escuela y la construcción de estrategias de cultura de paz. La escasez de estudios y la dificultad para comprender el tema, así como la importancia de integrar la educación y la promoción de una cultura de paz, fueron aspectos que derivaron en la sugerencia de construir un juego de mesa para apoyar la resolución de conflictos en un contexto no violento. El estudio señaló contribuciones a la promoción de la Cultura de Paz y estrategias lúdicas para el contexto de la Educación Infantil, así como la necesidad de realizar más investigaciones para explorar el tema.

Palabras clave: Derechos humanos; Crianza del niño; Cultura de paz.

\section{Introdução}

A cultura da paz baseia-se em valores humanos que precisam ser colocados em prática, transformando-se, concretamente, em atos. Perpassa as ações que se traduzem em aspectos éticos, morais e estéticos, compreendendo o envolvimento de crianças e adultos com os princípios do respeito, liberdade, justiça, democracia, direitos humanos, tolerância, igualdade e solidariedade, nos diversos contextos sociais (Santos \& Garcez, 2019).

A Cultura de Paz converge para uma metodologia que busca propagar um conjunto de valores, atitudes, tradições, comportamentos e estilos de vida baseados no respeito à vida, no fim da violência e na promoção e prática da não-violência por meio da educação, diálogo e cooperação. Desse modo, a paz não é entendida somente pela ausência de conflitos, mas como um processo positivo, dinâmico e participativo que promove o diálogo na resolução dos conflitos em um espírito de entendimento e 
cooperação mútuos entre as pessosa, enfretando a injustiça e a intolerância, e rejeitando toda forma de discriminação e ódio (Soares \& Teixeira, 2018).

Sua importância é reforçada no contexto escolar por intermédio do Plano Nacional de Educação, que busca garantir a existência de políticas de combate à violência na escola, inclusive pelo desenvolvimento de ações destinadas à capacitação de educadores para detecção dos sinais de suas causas, como a violência doméstica e sexual, favorecendo a adoção das providências adequadas para promover a construção da cultura de paz e um ambiente escolar dotado de segurança para a comunidade (Assis \& Ribeiro, 2017; Brasil, 2014).

A qualidade de ensino é permeada pela qualidade das relações pessoais entre os atores envolvidos. No ambiente escolar, essa influência é fundamental para que ocorra o processo de ensino e aprendizagem. Desse modo, para o alcance de resultados de qualidade em um ambiente que seja embasado em violência, é necessário o reforço e adesão aos princípios anteriormente citados (Perdomo, Leal \& Carmo, 2018).

A escola é um espaço social favorável ao desenvolvimento de atitudes e valores, todavia, não se pode despertá-los nos educandos se esses não forem pessoas capazes de compreender e ajudar o outro e, da mesma forma, se seus planejamentos pedagógicos não estiverem embasados nos preceitos de uma cultura para a paz, imprescindíveis na atualidade (Ribeiro, Ribeiro \& Tunice, 2018).

A educação para a paz constitui uma estratégia relevante que contribui para uma infância e sociedade mais humanizada. Para fomentar sua promoção é importante a compreensão das relações que violência e paz apresentam com as condições políticas, econômicas, de educação e organização social, visto que são aprendidas culturalmente (Dimas, 2018).

Com base nas experiências das autoras em atividades de ensino, pesquisa e extensão em espaço social escolar de grande vulnerabilidade, buscou-se responder à questão da pesquisa: "Qual a percepção de educadores sobre estratégias de cultura de paz na educação infantil?”. Assim, tem-se por objetivo conhecer a percepção de educadores sobre estratégias de cultura de paz na educação infantil.

\section{Metodologia}

Esse artigo foi extraído da pesquisa "Aplicação de estratégias para cultura da paz na educação infantil: Percepção de educadores", aprovado pelo Programa Institucional de Bolsas de Iniciação Científica (PIBIC), ciclo 2019-2020, da Universidade Federal de Alagoas, registrado pelo CAAE: 18038619.5.0000.5013. É um estudo de abordagem qualitativa, realizado por meio da tecnologia de grupos focais (GF), em que os pesquisadores fizeram a coleta de dados qualitativos por meio de entrevistas grupais, tendo como participantes os profissionais da educação (Silva, Castro-Silva \& Moura, 2018; Schvingel, Giongo \& Munhoz, 2017).

A coleta de dados ocorreu através das sessões do GF, onde os profissionais fizeram o relato das percepções sobre as atividades no eixo temático de Cultura de Paz com as crianças situadas na primeira infância ( 0 a 6 anos de idade), no período de dezembro de 2019 a março de 2020. Os participantes do estudo foram os profissionais de educação (professoras, auxiliares e coordenadoras) do Centro Municipal de Educação Infantil Heloísa Marinho de Gusmão, localizado no bairro Benedito Bentes, cidade de Maceió - AL.

O grupo focal é um método de coleta de dados em que os pesquisadores realizam a coleta de dados qualitativos por meio de entrevistas grupais. Essa modalidade é apropriada para estudos que buscam entender atitudes, preferências, necessidades e sentimentos. Neste ordenação, um grupo de pessoas é reunido pelo pesquisador a fim de discutir e comentar um tema e o objeto da pesquisa, a partir de experiências vividas acerca de assuntos emergentes na sociedade e problematizadas pelo investigador (Schvingel, Giongo \& Munhoz, 2017). 
A descrição do percurso metodológico da pesquisa em que foram inseridos os grupos focais (GF) é discutida na seção intitulada percurso metodológico. Na seção seguinte, resultados e discussão, buscou-se apresentar os dados referentes à aplicação da tecnologia de GFs em três subseções, como categorias que emergiram mediante a realização da pesquisa. O GF apresenta várias vantagens, como oportunizar ao pesquisador o conhecimento de "atitudes, comportamentos e percepções dos pesquisados 'in loco' além de reduzir os custos e o tempo gasto se comparado com outras técnicas e permitir, ainda, que se obtenha para análise um material que não surgiria em uma conversação casual, tampouco em resposta a perguntas previamente formuladas pelo investigador (Schvingel, Giongo \& Munhoz, 2017; Santos et al., 2019).

A partir disso, foi constituído um grupo segundo os seguintes critérios: nove profissionais da educação que lidem com crianças na primeira infância, instrumentalizado por guia temático focado em três objetivos principais, sendo cada um o foco de uma sessão e a ambientação nos locais de estudo para recrutamento dos participantes, de forma a mantê-los confortáveis durante a sua realização. Para a preservação da identidade dos participantes da pesquisa, todos foram identificados conforme sistema alfanumérico, utilizando a letra "P", seguido de um numeral, que correspondia a posição de fala. As falas foram transcritas na íntegra e os dados produzidos foram analisados e interpretados por meio da análise de conteúdo temática.

\section{Resultados e Discussão}

As etapas de planejamento do GF foram imprescindíveis para a obtenção de resultados efetivos. Cada uma das discussões seguiu a mesma metodologia: um resgate sobre a temática era feito de maneira simples pelas pesquisadoras e, a seguir, uma pergunta norteadora iniciava a discussão do tema. Durante todo o processo, as pesquisadoras se mantiveram imparciais, fazendo apenas os questionamentos necessários na tentativa de não incentivar respostas ou relacionar-se com elas. Foram realizadas quatro sessões com duração de 60 minutos cada, que ocorreram em uma sala localizada no ambiente escolar (e local de trabalho dos profissionais), em datas e horários acordados com os participantes.

O primeiro encontro do GF (Figura 1) realizado em dezembro de 2019, teve por objetivo colher as assinaturas do Termo de Consentimento Livre e Esclarecido (TCLE), apresentar os objetivos da pesquisa e fazer uma conceituação a respeito da cultura de paz. O segundo encontro do GF, realizado em janeiro de 2020, teve por objetivo compreender o que os profissionais entendem por cultura de paz. No terceiro encontro do GF, realizado em fevereiro de 2020, objetivou-se colher os relatos de experiência a respeito das atividades realizadas em sala de aula sobre a temática de Cultura de paz. Na quarta e última sessão, realizada em março de 2020, foram fomentadas reflexões a respeito das temáticas importantes e que deveriam estar presentes em jogos e brincadeiras voltados para a cultura de paz.

Figura 1: Divisões dos grupos focais mediante seus objetivos.
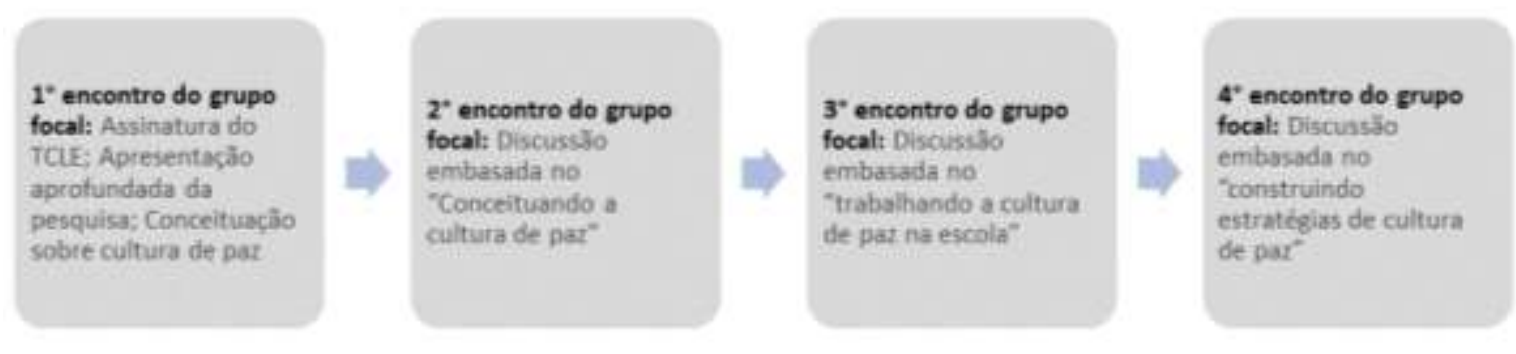

Fonte: Autores (2021). 
A recepção das percepções dos profissionais a respeito da cultura de paz e da utilização de suas metodologias foi conduzida a partir de roteiros de GF previamente sistematizados, com o objetivo de seguir uma mesma linha de construção e captação de reflexões. Como citado, tiveram o intuito de responder perguntas, elaboradas de forma a impossibilitar que os pesquisadores influenciassem, de alguma forma, em suas respostas. A partir dos quatro encontros realizados emergiram três categorias principais: conceituando cultura de paz, trabalhando a cultura de paz na escola e construindo estratégias de cultura de paz.

\subsection{Conceituando a cultura de paz}

O segundo encontro do GF foi iniciado com um breve resgate, pelas pesquisadoras, de conceitos sobre a cultura de paz, apenas para provocar as discussões. Nesse primeiro momento, buscou-se compreender qual a visão dos participantes do que seria a cultura de paz, individualmente. A partir da solicitação norteadora: "tentem falar, em poucas palavras, o que seria cultura de paz para vocês" foi dado início a coleta de dados da pesquisa. Um dos participantes iniciou as discussões abordando um conceito que se aproxima daquele apresentado pelas Organizações das Nações Unidas, em 1999, sendo ele:

"Cultura de paz, ao meu ver, poderia ser um movimento que conscientize, positivamente, valores e atitudes que são necessários na convivência entre pessoas e nações. As crianças têm uma imensa capacidade de compreender por meio do exemplo o que é certo e errado, tanto é que elas passavam a ensinar aos colegas, ajudando-se mutuamente, com trocas positivas". P1

Para a Organização das Nações Unidas, a cultura de paz é considerada um conjunto de valores, atitudes, tradições, comportamentos e estilos de vida baseados no respeito à vida, no fim da violência e na promoção e prática da não-violência por meio da educação, do diálogo e da cooperação (...), bem como na adesão aos princípios de liberdade, justiça, democracia, tolerância, solidariedade, cooperação, pluralismo, diversidade cultural, diálogo e entendimento em todos os níveis da sociedade e entre as nações (ONU, 1999). A fala do participante reforça e abarca esses conceitos. A discussão trouxe o entendimento de cultura de paz atrelado à transmissão de "bons valores" para as crianças, bem como do papel importante da escola frente a este processo.

"Cultura de paz, como outros valores, tem que ser passado na prática. Praticando em sala de aula tolerância, amor, muito amor e carinho, respeito. Se porventura a criança vem com agressividade, nós devolvemos com carinho. Não é fácil e nem se resolve do dia pra noite. Mas é possivel... Um trabalho de formiguinha. Na escola no geral. No ambiente escolar." P3

O "trabalho de formiguinha" citado na fala do participante é uma realidade bastante comum ao se tratar da promoção da cultura de paz na sociedade. Para abordá-la com as crianças, é importante que ela seja trabalhada e incentivada, primeiramente, entre os profissionais, para que seus conceitos sejam repassados da melhor forma possível (Fernández, Guzmán, Gámez \& Casado, 2019). Além disso, é notório que se vejam oportunidades para utilizar dos conceitos da cultura de paz continuamente, principalmente porque as crianças tendem a reproduzir as situações que vivenciam:

"Acredito que a cultura de paz deve ser trabalhada com as crianças, os valores e sentimentos bons, através de diálogos e atitudes positivas, porque as nossas crianças reproduzem muito em sala a violência que elas vivenciam." P4 
A discussão a respeito do papel da escola na promoção de uma cultura de paz foi iniciada e a abordagem dessa percepção é extremamente relevante, visto que é um dos ambientes em que a criança é incentivada a expressar seus sentimentos, conviver com as diferenças, bem como aprender a como solucionar os conflitos que lhe são apresentados e se esse ambiente é acolhedor, maior é a possibilidade de a criança aprender o que está sendo ensinado (Arenas-Villamizar, Mora-Wilches \& Medina-Mendoza, 2019):

\section{“Devemos demonstrar que a escola é um espaço seguro e acolhedor.” P4}

Outras definições a respeito dos "bons valores" foram sendo apresentadas ao longo do GF, o que levou as pesquisadoras a buscarem compreender o que essa expressão significaria e de que forma influencia as crianças. Algumas falas foram destacadas:

\footnotetext{
“No meu ver cultura de paz são os bons valores, pois estes podem consolidar na criança boas condutas. ” P7

"Acredito que a cultura de paz deve ser trabalhada com as crianças, os valores e sentimentos bons, através de diálogos e atitudes positivas." $\mathrm{P} 4$

"É se opor a violência e incentivar o respeito." $\mathrm{P} 9$
}

Para os educadores, a busca pela cultura de paz e o ensino de seus conceitos às crianças objetiva ensiná-los a respeitar o próximo, evitar práticas violentas, e, principalmente, incentivar os bons sentimentos. A comunicação não violenta abarca essas exemplificações. Essa, é tida como uma abordagem para expor suas necessidades e seus pensamentos em consonância com as necessidades do outro, de maneira autêntica e desarmada. Por meio da sua utilização, se evitam conflitos e julgamentos sobre o que seria mais correto ou não, possibilitando a imposição das opiniões de todos os sujeitos envolvidos (Santos, 2018). Durante a discussão, outro questionamento importante foi elencado pelas pesquisadoras, sendo ele: "Para você, por que é importante se trabalhar a cultura de paz nessa faixa etária?". Algumas das educadoras apontaram que:

"Para termos um ambiente acolhedor e seguro para a criança, possibilitando um pleno desenvolvimento físico, emocional e social. Possibilitando a interação social e o respeito mútuo, num ambiente que valorize a paz." P5

"Nessa idade elas já têm uma compreensão melhor do que se é falado. Já entende. E acabam sendo agentes da paz em suas casas levando o aprendizado do ambiente escolar." P3

Durante o encontro, alguns participantes se mantiveram em silêncio ou reforçaram a fala da participante anterior, mas sem formular o seu próprio conceito. Essa situação pode sinalizar para algumas impressões, como o possível desconhecimento do conceito da cultura de paz, bem como a sua dificuldade de compreensão. Tal vivência reforçou a importância de sensibilizar esses profissionais ao aprofundamento sobre a temática, visto que a perpetuação da não violência é fundamental para o sucesso de ações de cultura de paz que, para as crianças, tem como referência, principalmente, os centros educacionais e os profissionais da educação (de Sousa, 2019).

A educação para a paz encontra nos centros educacionais um ambiente privilegiado para desenvolver seu trabalho e é um direito de cidadania das crianças. Além disso, o ambiente escolar é gerador de contextos e ações de mediação e formação que permitem o desenvolvimento da sociabilidade e da circulação social, bem como a promoção cultural para alargar as 
perspectivas educacionais, de trabalho, de lazer e de participação social, tornando extremamente necessário que esses profissionais compreendam o importante papel que possuem (del Mar Garcia-Vita, Anãños \& García, 2020).

\subsection{Trabalhando a cultura de paz na escola}

No penúltimo GF, buscou-se colher relatos de experiência a respeito das práticas realizadas em sala de aula sobre a temática da Cultura de paz, bem como fomentar reflexões a respeito das atividades mais aceitas pelas crianças. Teve como questão norteadora: “Atividades referentes à cultura de paz foram realizadas em sala de aula? Qual a aceitação?”. A discussão teve início com a citação da utilização das histórias infantis e isso é fortemente comum, visto que é uma atividade fundamental para transmitir conhecimentos e valores; sua atuação é decisiva na formação e no desenvolvimento do processo ensinoaprendizagem (Sousa Machado, da Silva \& Silva, 2021).

“Nas histórias infantis. De alguma forma falando em respeito ao próximo, amor, união, tolerância.” P1

A contação de histórias é uma metodologia muito presente no contexto educativo voltado para a criança. Sua utilização é validada em razão de possibilitar à criança, no processo de sua maturação, o desenvolvimento da criatividade e senso-crítico, relevante ao desenvolvimento cognitivo; aquisição de conhecimento acerca do mundo que a cerca e das culturas e práticas presentes nele e, no contexto da cultura de paz, acerca da sua utilização, princípios e importância (Silva, 2017; Gonçalves \& Rodrigues, 2019). Um dos participantes reforçou a exemplificação anterior, citando outras práticas utilizadas por ele, e reforçou um aspecto importante vivenciado por muitos profissionais da educação:

"Minha experiência é trabalhar a cultura de paz usando o que eles mais gostam... contação de estórias, jogos, músicas, dinâmicas e no dia a dia mesmo, tendo que intermediar os conflitos entre eles. Não acho difícil trabalhar cultura de paz com as crianças... Dificil é conscientizar alguns pais. Muitas vezes o trabalho que fazemos é desfeito quando eles chegam em casa." P3

Atividades voltadas para a motricidade também são realizadas, possibilitando a associação entre o estímulo ao desenvolvimento infantil com o ensino a conceitos de cultura de paz:

"Na primeira semana de aula as crianças pintaram plaquinhas com gravuras de como respeitar os colegas. Trabalhamos muito a questão do respeito através da roda de conversa no dia a dia e nas brincadeiras. As crianças aceitaram bem, elas sempre interagem com o grupo." $\mathrm{P9}$

A motricidade faz parte das dimensões envolvidas nas etapas do desenvolvimento infantil e é caracteriza-se como o processo de aquisição de habilidades neuropsicomotoras, cognitivas, sociais e afetivas, importantes para a autonomia e maturação da criança até a vida adulta. É influenciado por fatores próprios do organismo infantil, mas também é extremamente sensível ao mundo externo, capaz de estimular sua ocorrência de maneira positiva ou negativa (Morais, Carvalho \& Magalhães, 2016).

Dado esses conceitos, ressalta-se que a experienciação de realidades violentas é considerada um tipo de estímulo externo negativo e de grande impacto ao desenvolvimento infantil, podendo interferir tanto na criança, ressaltando a importância de ter a cultura de paz como estratégia de prevenção a esses efeitos (Hino et al., 2019; Grasseli, 2017). A proposta de se trabalhar a cultura de paz nas atividades simples do cotidiano das crianças são consideradas pelos profissionais, como na fala de uma participante: 
"No dia a dia nos deparamos com situações e conflitos entre as crianças e já é uma oportunidade para inserir a “cultura de paz.” P1

No entanto, poucas foram as exemplificações de atividades, apesar de justificarem a facilidade de se trabalhar a temática. Outros profissionais, todavia, justificaram a não realização em razão da faixa etária que possuem contato (menores de 4 anos de idade). Entretanto, mesmo com as crianças menores é possível que se busque incentivar uma cultura de paz, como é reforçado na fala:

"Sim, realizei atividades referentes ao tema e foram muito bem aceitas pelas crianças. Acho que trabalhamos a cultura de paz diariamente quando orientamos as crianças a respeitarem seus colegas, a tratarem bem e com educação a todos que fazem parte da escola, enfim, são nas atitudes simples do dia a dia." P7

$\mathrm{Na}$ fase pré-escolar, contexto de trabalho dos educadores, a recreação é fortemente presente no processo de desenvolvimento dessas crianças e, com as falas apresentadas, sugere-se a busca pelo entendimento sobre de que maneira é possível a construção da cultura de paz com atividades que se enquadrem à faixa etária, principalmente de crianças menores. Dessa maneira, é relevante compreender a junção entre as atividades voltadas à recreação e ao processo educativo; de obtenção de conhecimento e autonomia, bem como da resolução de conflitos vivenciados na faixa etária (Grasseli, 2017). Buscou-se compreender, também, se esses educadores passavam por dificuldades para a realização de atividades voltadas para cultura de paz nessa faixa etária (de 4 a 6 anos de idade). O que percebeu-se por meio das falas:

"Não. As crianças são muito abertas à cultura de paz. Elas são exemplo de amor, união, perdão. Às vezes, a dificuldade surge quando elas vivenciam uma realidade de violências. Fica difícil assimilar o que passamos em sala se na maior parte do tempo a realidade delas é contrária. Mas no geral, elas se mostram abertas a essa cultura de paz.” P3

"Não. Porque, já trabalho no dia a dia, ensinando os valores e explicando que temos direitos, deveres e que todos somos responsáveis pelo mundo que nos cerca. E as crianças têm demonstrado através de pequenos atos de carinho entre os colegas." P5

Apesar das falas, verifica-se que muitos profissionais da educação ainda possuem dificuldades para compreensão sobre a cultura de paz e para pautar e adaptar suas atividades escolares nessa temática de maneira que as crianças aprendam o que está sendo instruído. Isso, justifica-se segundo alguns autores na falha da comunicação intraescolar, desmotivação, falta de preparo dos profissionais frente a temática e, muitas vezes, na não inclusão da mesma nos projetos pedagógicos (Lopes et al., 2019).

Outros profissionais justificaram a não realização de atividades voltadas para a cultura de paz mediante a impossibilidade frente a faixa etária em que trabalham. Todavia, a cultura de paz pode ser exercitada de maneira simples, na busca do respeito entre as crianças e as suas diversidades e na mediação de conflitos existentes no dia a dia e isso pode ser realizado em todas as faixas etárias, principalmente com crianças pequenas (Amaral \& Ramos, 2018; Lopes et al., 2019).

\subsection{Construindo estratégias de cultura de paz}

No quarto e último GF buscou-se identificar subtemáticas de cultura de paz relevantes a serem trabalhadas com crianças, para utilizá-las como estratégias na elaboração de atividades. Uma das participantes mostrou-se confusa, mas relatou:

"Essa pergunta me deixou confusa, não sei se isso responde, mas acho que um dos temas a ser abordado são os direitos e deveres. Trabalhei isso quando estabeleci as regras da sala no início do ano letivo.” P7 
A mesma participante citou, ainda, uma realidade muito comum no cenário em que a instituição está inserida: o preconceito. Sua fala ressaltou a importância da inclusão da temática em jogos e brincadeiras:

"Sim, também, e o preconceito racial, pois no ano passado eu tinha um aluno que tratava o colega mal por ser negro, e eu sempre conversava muito sobre isso, não sei se consegui fazê-lo esquecer esse preconceito, mas isso me fez ver que o preconceito é mais presente do que eu imaginava. Então sempre que tenho oportunidade falo sobre isso com minha turma. " $\mathrm{P7}$

A fala da participante introduz o conceito de violência racial, também conhecido como discriminação étnico-racial, que faz parte da realidade das crianças em todas as faixas etárias. Por discriminação étnico-racial compreende-se toda distinção, exclusão, restrição ou preferência baseada na cor, raça ou descendência e que tenha por objetivo anular ou restringir o exercício pleno dos direitos humanos (Brasil, 2010).

Por estar enraizada na sociedade, seus conceitos adentram a realidade das crianças, acarretando em atos de reprodução daquilo que é vivenciado, justificados em sua imaturidade em compreender a temática de maneira extensiva. Os resultados trazidos por esse tipo de agressão refletem, muitas vezes, na vida adulta, causando traumas e atrasos em seu desenvolvimento. Tais justificativas reforçam a importância de se tratar o respeito ao diferente desde os primeiros anos de vida (Cardoso \& Pazo, 2017).

As demais participantes foram mais pontuais em suas sugestões, trazendo as temáticas de: "amor, família" (P2); "empatia“" (P1); "respeito" (P6); "esperar a vez" (P1); "solidariedade" (P5); "paciência” (P4); "crianças que não aceitam perder em jogos" (P4); "trabalho em equipe, empatia" (P2); "Respeito, honestidade e solidariedade" (P9); "jogo para trabalhar os sentimentos" (P4); "a paz, a família, o amor, o respeito mútuo, o respeito a natureza, solidariedade” (P8). Uma das participantes referiu a temática da violência, pontuando que ela é presente na realidade das crianças:

"Mesmo de forma tímida já podemos observar algumas questões sobre preconceito, violência por meio de gestos e palavras." $\mathrm{P} 1$

Essa fala reforça a importância da escola como um todo estar capacitada e preparada para a detecção de sinais de violências as quais a criança pode estar sujeita, pois ela pode ser capaz de refletir e interferir no percurso normal de seu crescimento e desenvolvimento, atrasando-o (Egry, Apostólico, Morais \& Lisboa, 2017). Outra participante pontuou a respeito de um momento muito comum no dia a dia das crianças e que pode ser aproveitado para se abordar a cultura de paz em jogos e brincadeiras:

"Falando na hora do lanche, há crianças que gostam de comer um pouquinho do lanche do outro mas não gosta de partilhar o seu." $\mathrm{P} 3$

A mediação de conflitos diários, dos simples aos mais complexos, são considerados momentos importantes para se ensinar a respeito da cultura de paz e também podem ser utilizados em jogos e brincadeiras (Alves, 2019). Também surgiu a discussão a respeito da temática de ideologia de gênero e pluralidade familiar, e como o questionamento da sua elegibilidade e, se deve ser trabalhado nessa idade. Uma das participantes pontuou: 
"Acho que para as crianças ainda não existe esse conflito de gêneros. Já em relação a Pluralidade Familiar algumas crianças já vivenciam situações homoafetivas, adotivas, etc, não sei como esse tema seria abordado e entendido por eles." P2

Apesar de tratar de uma temática relevante e atual, poucas pesquisas foram realizadas com enfoque na adoção da cultura de paz no contexto escolar, principalmente na primeira infância. Percebeu-se a escassez de sua abordagem na educação infantil, e também a dificuldade dos participantes em opinar a respeito, relacionado aos momentos de silêncios vivenciados nos grupos focais. Apesar desses casos, compreende-se que a adoção da temática constituiu um gatilho para a sua adoção pelos educadores, assim como para outros profissionais da educação e pesquisadores busquem compreendê-la e inseri-la em seu contexto de trabalho.

O efeito de sua importância foi comprovado pela sugestão, por parte dos participantes, na construção de um jogo de tabuleiro educativo voltado para a abordagem da temática de maneira de fácil compreensão por crianças em idade escolar. Durante o jogo, as crianças terão oportunidade de vivenciar experiências sobre diversos temas e terão que resolver os pequenos conflitos utilizando os princípios da cultura de paz.

\section{Conclusão}

A escolha da proposta dessa pesquisa impactou de maneira positiva tanto profissionais de educação quanto as pesquisadoras. As discussões possibilitaram a quebra da visão cômoda a respeito da cultura de violência, para servir de gatilho na produção de ações que possam servir no seu combate e no incentivo à uma cultura voltada para a paz, entendimento da pluralidade e redução de preconceitos.

Foi possível compreender que os profissionais da educação, mesmo conhecendo a importância da cultura de paz, não possuem uma ideia ampla de sua relevância e de seus conceitos, demonstrando a necessidade de discutir este assunto com mais propriedade entre profissionais de educação para que assim possa ser explorado e trabalhado na escola e, consequentemente, influenciar na transformação social das famílias.

As discussões também foram importantes para reforçar que uma sociedade construída para incentivar a paz deve ter início nos primeiros anos de vida, com as crianças: seja na transmissão de valores ou no ensino a resolução de conflitos de maneira harmônica, utilizando metodologias apropriadas para cada faixa etária.

Com a realização deste estudo foi possível concluir que o conhecimento a respeito da cultura de paz ainda é escasso e seu incentivo é de extrema importância para a construção de uma sociedade voltada à não violência desde a primeira infância. Também sugere-se a realização de mais pesquisas que explorem o entendimento da Cultura de paz por parte dos profissionais de educação, bem como sua aplicação em meio escolar, visto que até para a construção da temática, houve certa escassez em materiais de apoio.

\section{Agradecimentos}

Nossos agradecimentos são destinados à Universidade Federal de Alagoas (UFAL) e ao Conselho Nacional de Desenvolvimento Científico e Tecnológico (CNPq) pela possibilidade do desenvolvimento do estudo e pelo seu financiamento. Agradecemos, também, ao Centro Municipal de Educação Infantil Heloísa Marinho de Gusmão que sempre se encontra aberto ao desenvolvimento de nossas pesquisas. 


\section{Referências}

Alves, A. F. (2019). Reflexões sobre a cultura de paz na escola. Revista Com Censo: Estudos Educacionais do Distrito Federal, 6(3), 94-103. http://www.periodicos.se.df.gov.br/index.php/comcenso/article/view/648

Amaral, D. E. L. \& Ramos, J. F. P. (2018). Mediação de conflitos no ambiente escolar para promover a cultura de paz. Conhecer: debate entre o público e o privado, $8(21)$, 24-44. https://doi.org/10.32335/2238-0426.2018.8.21.1072

Arenas-Villamizar, V. V., Mora-Wilches, K. Y. \& Medina-Mendoza, L. A. (2019). Familia y escuela como entidades fundamentales en la construcción de paz. Aibi revista de investigación, administración e ingeniería, 7(s1), 24-31. http://dx.doi.org/10.15649/2346030X.495

Assis, V. M. S. \& Ribeiro, S. L. S. (2017). Professores e práticas pedagógicas para combater a violência e construir a cultura de paz. Atos de Pesquisa em Educação, 12(1), 166-188. http://dx.doi.org/10.7867/1809-0354.2017v12n1p166-188

Brasil (2014). Lei Federal n 13.005, de 25 de junho de 2014. Aprova o Plano Nacional de Educação - PNE e dá outras providências. Brasília, DF: Presidência da República. http://www.planalto.gov.br/ccivil_03/_ato2011-2014/2014/lei/113005.htm

Brasil (2010). Lei Federal n ${ }^{\circ}$ 12.288, de 20 de julho de 2010. Institui o Estatuto da Igualdade Racial; altera as Leis nº 7.716 , de 5 de janeiro de 1989, 9.029, de 13 de abril de 1995, 7.347, de 24 de julho de 1985, e 10.778, de 24 de novembro de 2003. Brasília, DF: Presidência da República. http://www.planalto.gov.br/ccivil_03/_ato2007-2010/2010/lei/112288.htm

Cardoso, V. C. \& Pazó, C. G. (2017). A violação dos direitos fundamentais das crianças e a reprodução da violência simbólica através dos contos de fada. Revista Direitos Humanos e Democracia, 5(10), 21-45. https://doi.org/10.21527/2317-5389.2017.10.21-45

de Sousa, N. H. B. (2019). As pesquisas para a Paz e os Direitos Humanos e a promoção da Cultura de Paz no ambiente escolar. Revista Com Censo: Estudos Educacionais do Distrito Federal, 6(3), 11-16. https://doi.org/10.5965/2358092521212019045.

del Mar García-Vita, M., Añaños, F. T., \& García, M. M. (2020). Educación social escolar en la construcción de cultura y educación para la paz: propuestas metodológicas de intervención socioeducativa. Campos en Ciencias Sociales, 8(2), 47-71. https://doi.org/10.15332/25006681/6012.

Dimas, S. (2018). A ética social e ambiental como fator cultural de humanização. International Journal of Philosophy and Social Values, 1(2), 103-121. https://doi.org/10.34632/philosophyandsocialvalues.2018.2712

Egry, E. Y., Apostólico, M. R., Morais, T. C. P. \& Lisboa, C. C. R. (2017). Enfrentar a violência infantil na Atenção Básica: como os profissionais percebem?. Revista Brasileira de Enfermagem, 70(1), 119-125. http://dx.doi.org/10.1590/0034-7167-2016-0009

Fernández, S. S., Guzmán, V. P., Gámez, T. R. \& Casado, R. R. (2019). La cultura de paz y conflictos: implicaciones socioeducativas/The culture of peace and conflicts: socio-educational implications. Collectivus, 6(1), 235-250. http://dx.doi.org/10.15648/Coll.1.2019.13

Gonçalves, J. R. \& Rodrigues, M. L. C. (2019). Cultura de Paz na Escolinha de Artes: diálogos necessários. Revista NUPEART, 21, 44-66. https://doi.org/10.5965/2358092521212019045

Grasselli, M. A. (2017). Educação infantil: respeitar a diversidade religiosa, superar as violências, educar para a paz: uma reflexão sobre as atividades interdisciplinares desenvolvidas no centro de educação infantil João Pedro de Aguiar. http://bdtd.faculdadeunida.com.br:8080/jspui/handle/prefix/39

Hino, P., Takahashi, R. F., Nichiata, L. Y. I., Apostólico, M. A., Taminato, M. \& Fernandes, H. (2019). As interfaces das dimensões da vulnerabilidade face à violência contra a criança. Revista Brasileira de Enfermagem, 72(suppl 3), 343-347. https://doi.org/10.1590/0034-7167-2018-0463

Lopes, M. F., Pontes, H. P., Oliveira, D. N., Melo, J. C., Ribeiro, J. E. M. \& Frota, M. A. (2019). Dificuldades intraescolares na efetividade do projeto de cultura de paz. Cogitare Enfermagem, 24, e62223.http://dx.doi.org/10.5380/ce.v24i0.62223

Morais, R. L. S., Carvalho, A. M. \& Magalhães, L. C. (2016). The environmental context and the child development: brazilian studies. Journal of Physical Education, 27, e2714. https://doi.org/10.4025/jphyseduc.v27i1.2714

ONU. Organização das Nações Unidas. (1999). Declaração e Programa de Ação sobre uma Cultura de Paz. Resolução aprovada por Assembléia Geral em 06 de outubro de 1999, $\quad \mathrm{n}^{\circ} \quad$ 53/243. http://www.comitepaz.org.br/download/Declara\%C3\%A7\%C3\%A3o\%20e\%20Programa\% 20de $\% 20$ A $\%$ C3\%A7\%C3\%A3o\%20sobre\%20uma\%20Cultura\%20de\%20Paz\%20-\%20ONU.pdf

Ribeiro, S. L. S., Ribeiro, M. T. M. \& Tunice, L. M. C. (2018). A Influência da Cultura e do Clima Escolar na construção e fortalecimento da Cultura de Paz. Educação, Cultura e Comunicação, 9(17), 309-320. http://unifatea.com.br/seer3/index.php/ECCOM/article/view/438/391

Santos, A. A., Pedreira, L. C., Freitas, R. A., Gomes, N. P., Menezes, T. M. O. \& Moura, L. V. C. (2019). Grupo focal como técnica de coleta de dados na pesquisa em enfermagem: Um relato de experiência. Revista Eletrônica Acervo Saúde, 11(17), e1648. https://doi.org/10.25248/reas.e1648.2019

Santos, F. S. \& Garcez, M. V. S. (2019). A cultura de paz, direitos humanos e educação para a paz em prol do desenvolvimento. Revista Caminhos-Revista de Ciências da Religião, 17(2), 590-605. http://dx.doi.org/10.18224/cam.v17i2.7312

Santos, M. A. S. C. (2018). A comunicação não violenta como instrumento para uma cultura de paz: uma proposta para as escolas da rede estadual de Sergipe. Ideias e Inovação-Lato Sensu, 4(2), 89-102. https://periodicos.set.edu.br/ideiaseinovacao/article/view/5611/2834

Schvingel, C., Giongo, I. M. \& Munhoz, A. V. (2017). Grupo focal: Uma técnica de investigação qualitativa. Debates em Educação, 9(19), 91-106. https://www.seer.ufal.br/index.php/debateseducacao/article/view/3455/pdf

Silva, F. M. S. V. (2017). A importância da contação de história na educação infantil. https://repositorio.ufpb.br/jspui/bitstream/123456789/4094/1/FM SVS19032018.pdf. 
Research, Society and Development, v. 10, n. 9, e11410917722, 2021

(CC BY 4.0) | ISSN 2525-3409 | DOI: http://dx.doi.org/10.33448/rsd-v10i9.17722

Silva, A. D., Castro-Silva, C. R., \& Moura, L. D. (2018). Pesquisa qualitativa em saúde: percursos e percalços da formação para pesquisadores iniciantes. Saúde e Sociedade, 27, 632-645. https://doi.org/10.1590/S0104-12902018172700

Soares, E. M. S. \& Teixeira, L. M. (2018). Práticas educativas e cultura de paz: articulando saberes e fazeres. Caxias do Sul, RS: Educs. https://www.ucs.br/site/midia/arquivos/ebook-praticas-educativas.pdf

Souza Machado, H., da Silva, S. M. P., \& Silva, J. E. (2021). Desenvolvimento infantil, educação e primeira infância: Histórias infantis como alternativa pedagógica. Research, Society and Development, 10(7), e4410716373-e4410716373. http://dx.doi.org/10.33448/rsd-v10i7.16373

Perdomo, J. E. H., Leal, R. S. L., \& Caro, O. (2018). Desarrollo de la empatía para mejorar el ambiente escolar. Educación y ciencia, (21), 217-244. https://revistas.uptc.edu.co/index.php/educacion_y_ciencia/article/download/9407/7854/ 